Wójcik Justyna, Obuchowska Aleksandra, Ozga Alicja, Standyło Arkadiusz, Obuchowska Karolina, Piotrowska Paulina. Polycystic ovary syndrome (PCOS) - risk factor, diagnostic and current treatment. Journal of Education, Health and Sport. 2020;10(9):556-560. eISSN 2391-8306. DOI http://dx.doi.org/10.12775/JEHS.2020.10.09.067

https://apcz.umk.pl/czasopisma/index.php/JEHS/article/view/JEHS.2020.10.09.067

https://zenodo.org/record/4043733

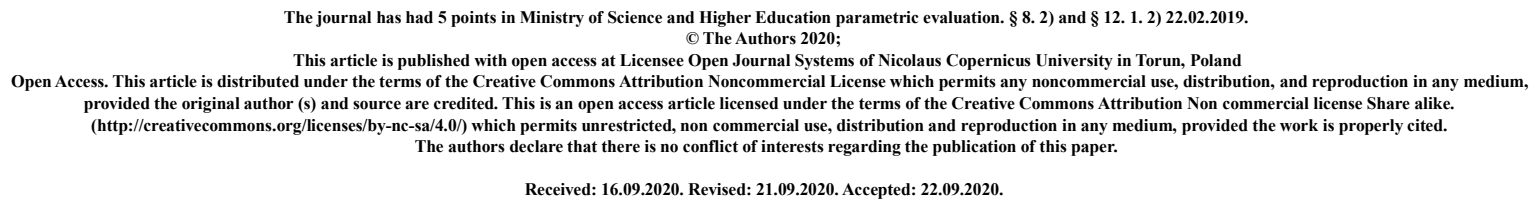

\title{
Polycystic ovary syndrome (PCOS) - risk factor, diagnostic and current treatment
}

\author{
Justyna Wójcik $^{1 \mathrm{a}}$, Aleksandra Obuchowska ${ }^{1 \mathrm{~b}}$, Alicja Ozga ${ }^{1 \mathrm{c}}$, Arkadiusz Standyło ${ }^{1 \mathrm{~d}}$, \\ Karolina Obuchowska ${ }^{1 e}$, Paulina Piotrowska ${ }^{1 f}$
}

ajustynawojcik455@gmail.com; https://orcid.org/0000-0001-7163-6784

baobuchowska12@gmail.com; https://orcid.org/0000-0003-0464-2695

caozga1@gmail.com; https://orcid.org/0000-0003-1291-905X

da.standylo@gmail.com; https://orcid.org/0000-0002-5154-4759

ekarolinaobuchowska99@gmail.com; https://orcid.org/0000-0003-4519-8236

fpaulina.piotrowska222@gmail.com; https://orcid.org/0000-0002-5516-952X

${ }^{1}$ Faculty of Medicine, Medical University of Lublin, Chodźki Street 19, 20-093 Lublin, Poland

Corresponding author:

Justyna Wójcik

Medical University of Lublin,

Chodźki Street 19

20-953 Lublin, Poland

e-mail: justynawojcik455@gmail.com

\section{Abstract \\ Introduction:}

Polycystic ovary syndrome (PCOS) is one of the most common endocrine and metabolic disorders in women in reproductive age, defined as a combination of signs and 
symptoms resulting from androgen excess and ovarian dysfunction in the absence of other specific diagnoses. PCOS is often associated with an increased risk of obesity, insulin resistance, metabolic disorders and cardiovascular events.

The aim of the study:

The purpose of this systemic review was to collect and analyze materials about risk factors, diagnostics, and the current treatment of polycystic ovary syndrome.

\section{Material and method:}

Review of the literature published in 2004-2018.

\section{Description of the state of knowledge:}

PCOS is characterized by an excessive secretion of androgens from the ovaries and / or adrenal glands. Intrinsic ovarian factors, such as altered steroidogenesis, and factors external to the ovary, such as hyperinsulinemia, contribute to the ovarian overproduction of androgens. PCOS requires careful selection of an appropriate diagnostic method and therapeutic approach to combat hyperandrogenism, a consequence of ovarian dysfunction and related metabolic disorders. Treatment of both women at risk of PCOS and those with a confirmed diagnosis of PCOS includes education, healthy lifestyle interventions and symptomatic therapeutic interventions.

\section{Summary:}

PCOS is a complex disorder involving many organs and affecting many body functions. It is important to diagnose this disease as soon as possible in order to implement appropriately selected therapeutic treatment aimed at eliminating disease symptoms and reducing the systemic consequences of hyperandrogenism in patients.

Key words: polycystic ovary syndrome, PCOS, diagnostics of PCOS, personalized therapy

\section{Introduction:}

Polycystic ovarian syndrome (PCOS) is a multifactorial and systemic disease, first described in 1935 by Stein and Levental[1]. The pathogenesis of PCOS has not been fully elucidated, but four aspects appear to contribute to the syndrome, including increased androgen secretion from the ovaries and / or adrenal glands, partial retention of folliculogenesis, insulin resistance, and neuroendocrine axis dysfunction. The final etiology remains unclear, but PCOS is recognized as having a strong genetic component [2]. This syndrome leads to infertility, insulin resistance, obesity, and cardiovascular problems, including many other health problems [3]. Obesity, lipid metabolism disorders, abnormal fibrinolysis and arterial hypertension often develop as early as adolescence [4]. 
Epidemiological studies have not shown increased mortality in women with PCOS, but the risk of developing cardiovascular diseases seems to be significant [5]. The hormonal imbalance caused by this syndrome leads to the appearance of cysts in the ovarian follicles. A cyst is a fluid-filled cyst that contains an egg cell that should normally be released into the lumen of the fallopian tube for the purpose of reproduction. The egg cell is transformed into a cyst, called a "functional cyst" [3].

The diagnosis of PCOS is still a matter of debate, but the absence or irregular periods, hyperandrogenism, and ultrasound of the ovaries are always taken into account.

Treatment is aimed at alleviating the symptoms of the disease, but it is also important to change the lifestyle, maintain an appropriate body weight by implementing a healthy diet and physical activity.

\section{The aim of the study:}

The purpose of the systemic review was to collect and analyze material of risk, diagnostics, and the current treatment of polycystic ovary syndrome.

\section{Material and method:}

Review of the literature published in 2004-2018.

\section{Discussion:}

The patogenesis of PCOS also includes patient's occupational exposure to chemicals. Not only pesticides, car exhaust fumes and environmental pollution contribute to the occurrence of PCOS, but also some hygiene and care products containing phthalates, parabens, isopropanol, glutaraldehyde, benzophenones or turpentine oil. It is important to consciously use cosmetic products and to choose those not containing harmful chemical compounds [6].

Symptoms that are a manifestation of PCOS include anovulation or oligovulation, an increase in androgen production that causes virilization or expression of male characteristics. The visible symptoms of hyperandrogenism include weight gain, abdominal and subcutaneous fat, hirsutism (facial and body hair), male pattern baldness (hair loss), clitoral enlargement (enlarged clitoris), deep voice, seborrhea (oily skin), and acne [7]. In addition to these morphological features, the metabolic profile changes. Insulin resistance is the main symptom of PCOS. It leads to hyperinsulinemia and may lead to diabetes [8]. PCOS patients often show increased carbohydrate appetite, frequent urination, delayed wound healing, fatigue, blurred vision, tingling sensation, mood swings, anxiety, and episodes of depression. These conditions are linked to diabetes, which can arise from PCOS. Patients often experience pelvic pain, fever, nausea, vomiting, urinary problems, constipation, etc. Pressing large cysts against the bladder or anus is responsible for abnormal urine flow and constipation [9]. In addition to observing physical symptoms, it is also worth paying attention to the possibility of psychiatric symptoms. Anxiety, depression, binge eating and bipolar disorder are observed as comorbidities with PCOS [10]. However, the range and severity of symptoms vary individually, so an individual approach to the patient is important. 
An early diagnosis of PCOS is important. The gynecologist makes the diagnosis taking into account the symptoms described by the patient and visible in the physical examination. The main criteria for PCOS were the criteria established in Rotterdam in 2003. In order to diagnose PCOS, two out of three criteria should be identified: amenorrhea or rare periods, clinical or biochemical hyperandrogenism, and the diagnosis of polycystic ovaries on an ultrasound image in group of adult women [11]. It is not necessary to visualize abnormal ovaries among adolescents.

The therapeutic management of PCOS may include oral contraceptive pills, which, taken for 6 months, alleviate hyperandrogenism and regulate menstrual cycles, inhibiting ovulation and preventing cyst formation [12]. Metformin, an insulin sensitizing substance that lowers the concentration of insulin and androgens, is also used in PCOS treatment [13]. Steroid hormone interventions are another way to solve PCOS problems. Aromatase inhibitors such as letrozole are being investigated for the induction of ovulation. Antiandrogens such as spironolactone, cyproterone acetate, flutamide, and finasteride are administered to treat androgenism [14].

All these treatments are effective to some extent, but not completely. Moreover, these treatments are not free from side effects. The importance and risk of therapy varies individually. Patient education about a healthy lifestyle also seems to be important. Patient should control the body mass index (BMI), use an appropriate calf, controlling the level of glycaemia. It is also important to encourage the patient to exercise regularly.

\section{Summary:}

PCOS is a serious multi-organ endocrinopathy that affects not only fertility but also the overall health of the patient. Although studies have shown that the impact of PCOS on life expectancy is negligible, the quality of life for women with this syndrome is significantly reduced. It is important to recognize this syndrome as early as possible, which reduces the intensity of unpleasant symptoms, significantly affecting well-being and mental health of women affected by this disease. Patients should also be aware of the fact that administered pharmacological therapy is only a part of the therapeutic success. It is also important to implement lifestyle improvements, maintain a constant, correct body weight and regular physical activity.

\section{Bibliography:}

1. Szydlarska D, Grzesiuk W, Bar-Andziak E, Controversies around pathogenesis polycystic ovary syndrome, 2010, 6(3): 141-146

2. Crespo, Raiane P, Bachega, Tania ASS, Mendonça, Berenice B, Gomes, Larissa G, An update of genetic basis of PCOS pathogenesis. Archives of Endocrinology and Metabolism 2018, 62(3):352-361.

3. Patel S, Polycystic ovary syndrome (PCOS), an inflammatory, systemic, lifestyle endocrinopathy, The Journal of Steroid Biochemistry and Molecular Biology, 2018, 182: 27-36

4. Barber T, Golding S, Alvey C, Global adiposity rather than abnormal regional fat distribution characterizes women with polycystic ovary syndrome, J. Clin. Endocrinol. Metab. 2008; 93: 999-1004. 
5. Meyer C, McGrath B, Teede H, Overweight women with polycystic ovary syndrome have evidence of subclinical cardiovascular disease, J. Clin. Endocrinol. Metab. 2005; 90: 5711- 5716.

6. Yang O, Kim HL, Weon JI, Seo YR, Endocrine-disrupting chemicals: review of toxicological mechanisms using molecular pathway analysis, J. Cancer Prev., 2015, 20: $12-24$

7. Madnani, Khan K, Chauhan P, Parmar G, Polycystic Ovarian Syndrome, Indian J. Dermatol. Venereol. Leprol., 2013, 79: 310 - 321

8. Sirmans SM, Pate KA, Epidemiology, diagnosis and treatment of polycystic ovarian syndrome, Clin. Epidemiol., 2013, 6: 1 - 13

9. Teede H, Deeks A, Moran L, Diamanti-Kandarakis, Kouli C, Bergiele A, Polycystic ovary syndrome: a complex condition with psychological, reproductive and metabolic symptoms that impacts health throughout life, BMC Med., 2010, 8: 41

10. Blay SL, Aguiar JVA, Passos IC, Polycystic ovary syndrome and mental disorders: a systematic review and exploratory meta-analysis, Neuropsychiatr. Dis. Treat., 2016, 12: $2895-2903$

11. T.R.E.-S.P.C.W.G.PCOS Consensus Workshop Group, Revised 2003 consensus on diagnostic criteria and long-term health risks related to polycystic ovary syndrome, Fertil. Steril., 2004, 8: 19-25

12. Piparva KG, Buch JG, Deep vein thrombosis in a woman using combined oral contraceptive pills,J.Pharmacol. Pharmacother. 2011, 2: 185 - 18

13. Naderpoor N, Shorakae S, Courten B, Misso ML, Moran LJ, Teede HJ, Metformin and lifestyle modification in polycystic ovary syndrome: a systematic review and meta-analysis,Szum. Reprod. Aktualizacja, 2015 , 21: 560 - 574

14. Davidson R, Motan T, Korownyk C, Clomiphene for anovulatory infertility, Mogą. Fam. Fiz., 2016, 62: 492 